ORIGINAL ARTICLE

\title{
Clinical evaluation of the COBAS Amplicor HBV monitor test for measuring serum HBV DNA and comparison with the Quantiplex branched DNA signal amplification assay in Taiwan
}

\author{
C-Y Dai, M-L Yu, S-C Chen, Z-Y Lin, M-Y Hsieh, L-Y Wang, J-F Tsai, W-L Chuang, W-Y Chang
}

J Clin Pathol 2004;57:141-145. doi: 10.1136/icp.2003.10835

See end of article for authors' affiliations

Correspondence to: Dr W-L Chuang, Hepatobiliary Division, Department of Internal Medicine, Kaohsiung Medical, University Hospital, No 100, ShihChuan 1st Road, Kaohsiung 807, Taiwan; d820195@ kmu.edu.tw, u8981103@kmu.edu.tw

Accepted for publication 17 July 2003

\begin{abstract}
Aims: To evaluate the performance characteristics and clinical usefulness of the COBAS Amplicor HBV monitor (COBAS-AM) test in Taiwan and to examine its correlation with the Quantiplex branched DNA signal amplification (bDNA) assay for measuring serum hepatitis $B$ virus (HBV) DNA concentrations. Methods: HBV DNA was measured by the COBAS-AM test in 149 sera from chronic HBV infected patients that had previously been analysed by the bDNA assay.

Results: The COBAS-AM test showed good reproducibility, with acceptable intra-assay and interassay coefficients of variation (1.6\% and $0.9 \%$, respectively) and good linearity $\left(r^{2}=0.98\right)$. The overall sensitivity of the COBAS-AM test was significantly higher than that of the bDNA assay $(95.3 \%$ v $83.2 \%)$ : $69.6 \%$ of samples with HBV DNA below the detection limit of the bDNA assay could be measured by the COBAS-AM test. There was a significant correlation between the results of the two assays $(r=0.901$; $p<0.0001)$. On average, the results derived from the COBAS-AM test were 0.55 log lower than those of the bDNA assay. HBV DNA concentrations were significantly higher among HBV e antigen (HBeAg) positive patients than negative ones, and higher among patients with abnormal alanine aminotransferase (ALT) concentrations than those with normal ALT concentrations ( $p=0.0003$ ).

Conclusions: The COBAS-AM assay, more sensitive in HBeAg negative samples than the bDNA assay, can effectively measure HBV DNA concentrations in Taiwanese patients. HBV DNA values measured by the COBAS-AM test and bDNA assay correlate significantly.
\end{abstract}

W th an estimated 350-400 million people worldwide infected chronically by hepatitis $\mathrm{B}$ virus (HBV), ${ }^{1}$ and the subsequent serious complications caused by liver damage including cirrhosis, liver failure, and hepatocellular carcinoma, HBV infection remains a global health issue. In Asian countries such as Taiwan, where HBV infection is mainly vertically transmitted and hyperendemic, HBV infection is the most important agent of chronic liver disease. ${ }^{2}$ Although numerous tests have been used to diagnose or monitor the course and outcome of HBV related liver diseases, direct detection of HBV DNA in serum is considered the most reliable method for diagnosing infection, particularly for some HBV genetic variations ${ }^{4}$ or occult HBV infection. ${ }^{56}$ Furthermore, quantitative measurement is valuable in predicting disease progression, monitoring HBV replication activity and infectivity, and assessing the response to antiviral treatment. ${ }^{78}$ Using the branched DNA signal amplification Quantiplex HBV DNA (bDNA; Bayer Diagnostics, Emeryville, California, USA) assay, HBV DNA was detected in $94-100 \%$ and $27-31 \%$ of hepatitis B e antigen positive and negative specimens, respectively, from chronic HBV infected patients. ${ }^{8}$ The bDNA assay may be useful as a prognostic and therapeutic monitoring tool for the management of HBV infected patients undergoing antiviral treatment. ${ }^{9}$

\footnotetext{
"Quantitative measurement is valuable in predicting disease progression, monitoring hepatitis B virus replication activity and infectivity, and assessing the response to antiviral treatment"
}

The first assays developed to measure HBV DNA were based on direct membrane hybridisation or solution hybridisation of a radiolabelled probe to the denatured strand of HBV DNA in serum..$^{10-14}$ Subsequently, non-radioactive hybridisation assays based on chemiluminescent probes were developed to overcome the disadvantages of using radioisotopes. ${ }^{10-15}$ The bDNA assay, which can detect HBV DNA at the lower limit of detection (LLOD) of $0.7 \times 10^{6}$ equivalents (eq) $/ \mathrm{ml}$, was reported to be better than other assays in measuring samples with low amounts of HBV DNA, ${ }^{8} 14$ 16 and was used as the standard for measuring HBV DNA in an antiviral trial. ${ }^{17}$ However, this assay is still not sensitive enough to detect the low viral load present in some patients, particularly those who respond to antiviral treatment. In addition, it was found that pronounced decreases in HBV DNA after antiviral treatment were related to better clinical outcomes and increases in HBV DNA during treatment, indicating rebound of viral replication, might predict the emergence of resistant variants. ${ }^{18} 19$ Thus, a more sensitive assay for the measurement of HBV DNA was needed for the evaluation of patients.

Methods for assessing HBV DNA by quantitative polymerase chain reaction (PCR) have been developed. ${ }^{720}$ The COBAS Amplicor HBV monitor test (COBAS-AM test; Roche

Abbreviations: ALT, alanine aminotransferase; bDNA, branched DNA signal amplification; COBAS-AM, COBAS Amplicor HBV monitor; eq, equivalent; $\mathrm{HBV}$, hepatitis $B$ virus; $\mathrm{HBeAg}$, hepatitis $B$ virus e antigen; $H B s A g$, hepatitis $B$ virus surface antigen; LLOD, lower limit of detection; $\mathrm{PCR}$, polymerase chain reaction 
Molecular Systems, Pleasanton, California, USA) is an automatic, PCR based assay for the measurement of HBV DNA in serum adapted for the automated COBAS Amplicor $^{\mathrm{TM}}$ system. $^{21}$ In our present study, HBV DNA concentrations were analysed retrospectively by the COBASAM assay in Taiwanese patients with chronic HBV infection. The performance characteristics and clinical usefulness of the COBAS-AM test were evaluated to assess the clinical sensitivity, reproducibility, and linearity of the COBAS-AM test and to examine the correlation between the COBAS-AM test and the bDNA assay for measuring serum HBV DNA values.

\section{METHODS}

\section{Patients and serum samples}

Between January 2000 and May 2001, serum samples from 149 patients with chronic hepatitis B, 132 males and 17 females, aged between 16 and 64 years (mean, 35.0; SD, 11.9) received by our laboratory consecutively were enrolled in our study. All patients were seropositive for HPV surface antigen (HBsAg) and were tested for HPV e antigen (HBeAg) by radioimmunoassay (Abbott Laboratories, North Chicago, Illinois, USA). All the serum samples were previously tested for HBV DNA by means of the bDNA assay, with the serum samples separated from whole blood collected in serum separation tubes and stored immediately at $-70^{\circ} \mathrm{C}$ in several aliquots. All of them were negative for antibodies to $\mathrm{HCV}$ using the third generation HCV antibody (anti-HCV) enzyme immunoassay test (Abbott Laboratories) and anti- $\delta$ by radioimmunoassay (Abbott Laboratories). Alanine aminotransferase (ALT) concentrations (normal upper limit of serum ALT, 34 IU/litre) were measured on a multichannel autoanalyser. Sera from three patients who were positive for anti-HCV antibodies and negative for HBsAg were used as controls in the HBV DNA assay.

\section{Measurement of serum HBV DNA}

HBV DNA was measured by two assays. HBV DNA had been measured previously in all the samples by means of the bDNA assay, according to the manufacturer's instructions. The range of HBV DNA measurements was $0.7 \times 10^{6}$ to $5700 \times 10^{6} \mathrm{HBV}$ DNA eq/ml. Measurement of HBV DNA by the COBAS-AM test was performed in this retrospective study according to the manufacturer's instructions. With four steps in the COBAS-AM test ${ }^{21}$ (specimen preparation, PCR amplification with biotinylated primers, hybridisation of the amplicons to specific oligonucleotide probes attached to magnetic particles, and colorimetric determination and quantitation of the amplicon-probe complex by measurement of the absorbance at $660 \mathrm{~nm}$ ) and a known concentration of a quantitative sample (QS) control included in all samples assayed, the absorbance measured is directly proportional to the amount of QS or HBV amplicon within the linear range of the assay. The dynamic range of the test was $2 \times 10^{2}$ to $2 \times 10^{5} \mathrm{HBV}$ DNA copies $/ \mathrm{ml}$. Serial dilutions were performed using the specimen dilution protocol provided by the manufacturer. Briefly, $25 \mu \mathrm{l}$ of serum from the patient was dispensed into $225 \mu \mathrm{l}$ of HBV DNA negative plasma (Basematrix; BM5-SP-534 Molecular; Boston Biomedica Inc, Boston, Massachusetts, USA) to make a $1 / 10$ dilution. A further $25 \mu \mathrm{l}$ of this $1 / 10$ dilution was then removed and dispensed into $225 \mu \mathrm{l}$ of HBV DNA negative plasma to make a $1 / 100$ dilution. Serial $1 / 1000,1 / 10000$, $1 / 100000$, and $1 / 1000000$ dilutions were made by repeating the dilution procedure. If the HBV DNA values were reported to be outside the linear range of the assay, the specimens were re-tested using a higher or lower dilution, or an undiluted sample, until the HBV DNA concentration could be measured.

\section{Reproducibility and linearity of the COBAS-AM test}

To investigate the reproducibility of the COBAS-AM test, three different samples with high, medium, and low viral loads were tested 10 times in one test run and then one time in each of five test runs. A separate aliquot of each sample was processed for each test so that the variations between results included those resulting from sample processing, amplification, and detection. To assess the linearity of HBV DNA measurement by the COBAS-AM test, two dilution panels were constructed by making serial threefold dilutions (in HBV negative serum) of high titre serum samples from patients who were positive and negative for HBeAg.

\section{Statistical analysis}

All HBV DNA results were expressed as mean (SD) after logarithmic transformation of the original values. The $\chi^{2}$ test with Yate's correction, Fisher's exact test, the Student's $t$ test, Spearman's rank correlation coefficient, simple linear regression, stepwise multiple linear regression, and multiple logistic regression were used. Results were deemed significant if $p$ was less than 0.05 .

\section{RESULTS}

\section{Performance characteristics of the COBAS-AM test}

Of the 149 patients, $78(47.7 \%)$ were positive for HBeAg and the other 71 (52.3\%) were HBeAg negative. The mean (SD) ALT concentration was 154.4 (256.0) IU/litre. The performance characteristics of the COBAS-AM test were analysed. Serum HBV DNA concentrations of three different samples with high, medium, and low titres were tested 10 times in the same round for intra-assay reproducibility, and the mean coefficient of variation (CV) was found to be $1.6 \%(2.0,1.9$, and $1.0 \%$, respectively; table 1$)$. Those tested five times in five different rounds for interassay reproducibility of the COBASAM test showed that the mean CV was $0.9 \%$ (1.3, 1.0, and $0.5 \%$, respectively; table 1$)$. These variations represented the total variability of the assay because a separate sample aliquot was processed for each test performed.

The linearity of HBV DNA measurement was assessed using dilution panels of two sera from patients positive and negative for HBeAg. The undiluted samples had viral loads of 7.79 and 7.29 logs, respectively, as determined by the COBASAM test. As shown in fig 1, the COBAS-AM assay showed a good linear response $\left(r^{2}=0.979\right.$ and 0.987 , respectively) in the threefold serial dilutions.

\section{Quantitative result of HBV DNA}

HBV DNA could be measured by the bDNA assay in 126 of the 149 patients $(84.6 \%)$. The concentration was higher than the upper limit of measurement in two of the $78 \mathrm{HBeAg}$ positive patients. In addition, HBV DNA could not be measured in $23(32.4 \%)$ of the $71 \mathrm{HBeAg}$ negative samples by means of the bDNA assay $(<0.7 \mathrm{Meq} / \mathrm{ml})$. However, using the COBAS-AM test, all 78 of the HBeAg positive samples were successfully measured. All 48 of the 71 HBeAg negative samples measurable by the bDNA assay could also be measured by the COBAS-AM test and 16 of the 23 samples with HBV DNA values below the LLOD for the bDNA assay could also be measured. The overall rate of HBV DNA measurement for the COBAS-AM test was $95.3 \%$, which was significantly higher than that for the bDNA assay $(83.2 \%$; $\mathrm{p}=0.002$ ). The two samples with HBV DNA values above the upper limit of the bDNA assay were measured successfully by the COBAS-AM test $\left(2.6 \times 10^{9}\right.$ and $2.9 \times 10^{9}$ copies/ $\mathrm{ml})$. The three control samples were not measurable by either the bDNA assay or the COBAS-AM test.

In the 124 specimens that could be measured by both the bDNA and COBAS-AM assays (23 low titre and two high titre samples for the bDNA assay excluded), there was a positive 
Table 1 Reproducibility of the COBAS-AM test

\begin{tabular}{|c|c|c|c|c|c|c|c|c|c|c|c|}
\hline Sera & $a$ & b & c & $d$ & e & $f$ & g & h & i & i & Mean (SD) \\
\hline \multicolumn{12}{|l|}{ Within run } \\
\hline Low titre & 6.82 & 6.86 & 6.75 & 6.67 & 6.62 & 6.53 & 6.71 & 6.51 & 6.47 & 6.53 & $6.65(0.14)$ \\
\hline Medium titre & 8.49 & 8.43 & 8.22 & 8.02 & 8.35 & 8.39 & 8.35 & 8.36 & 8.13 & 8.09 & $8.28(0.16)$ \\
\hline High titre & 9.05 & 8.91 & 9.04 & 8.98 & 9.03 & 8.96 & 8.87 & 8.79 & 8.87 & 8.96 & $8.94(0.09)$ \\
\hline \multicolumn{12}{|l|}{ Between run } \\
\hline Low titre & - & - & - & - & - & 6.63 & 6.69 & 6.58 & 6.49 & 6.70 & $6.60(0.01)$ \\
\hline Medium titre & - & - & - & - & - & 8.19 & 8.36 & 8.23 & 8.38 & 8.30 & $8.31(0.11)$ \\
\hline High titre & - & - & - & - & - & 8.99 & 8.92 & 8.92 & 8.88 & 9.01 & $8.95(0.05)$ \\
\hline
\end{tabular}

correlation between the two assays $(r=0.901$; $p<0.0001$; fig 2). Accordingly, the formula derived for the conversion of results is $\log$ (HBV DNA value by COBAS-AM test) $=$ $1.004 \times \log (\mathrm{HBV}$ DNA value by bDNA assay) -0.586 . On average, the results derived from the COBAS-AM test were $0.55 \log$ lower than the bDNA values (table 2). The mean differences between the two assays did not differ among samples that were positive or negative for HBeAg.

\section{The association between viral load and clinical characteristics}

HBV DNA could be measured in 142 patients (126 male and 16 female patients; age range, 16-64 years; mean, 34.9; SD, 12.0; 78 HBeAg positive; 64 HBeAg negative) by the COBASAM test. The mean (SD) HBV DNA and the mean (SD) ALT values were $6.84 \mathrm{l}$ (2.10) log copies/ml and 156.3 (259.1) IU/ litre, respectively, and the ALT concentrations of 123 patients $(86.6 \%)$ were abnormal. The association between HBV viral load and clinical characteristics of HBV infection was evaluated. The median HBV DNA concentrations of the HBeAg positive and HBeAg negative samples, as assessed by the CORBAS-AM test, were $10^{7.8}$ and $10^{6.9}$ copies $/ \mathrm{ml}$, respectively. Using univariate analyses, HBV DNA values were significantly higher in $\mathrm{HBeAg}$ positive than in $\mathrm{HBeAg}$ negative patients (mean, 8.04; SD, $1.19 v$ mean, 6.11; SD, 1.64 $\log$ copies/ml; $\mathrm{p}<0.0001$; fig 3), and higher in patients with an abnormal ALT concentration than in those with a normal ALT concentration (mean, 7.51; SD, $1.36 v$ mean, 6.04; SD, $2.43 \log$ copies/ml; $p=0.0003)$. Furthermore, the HBV DNA value correlated significantly with the serum ALT concentration $(p=0.03)$. No significant association between HBV DNA value and sex or age was observed. On multivariate analysis, HBeAg status and ALT concentration were associated with serum HBV DNA value $(\mathrm{p}<0.0001$ and $\mathrm{p}=0.01$, respectively). Serum HBV DNA value (not sex,

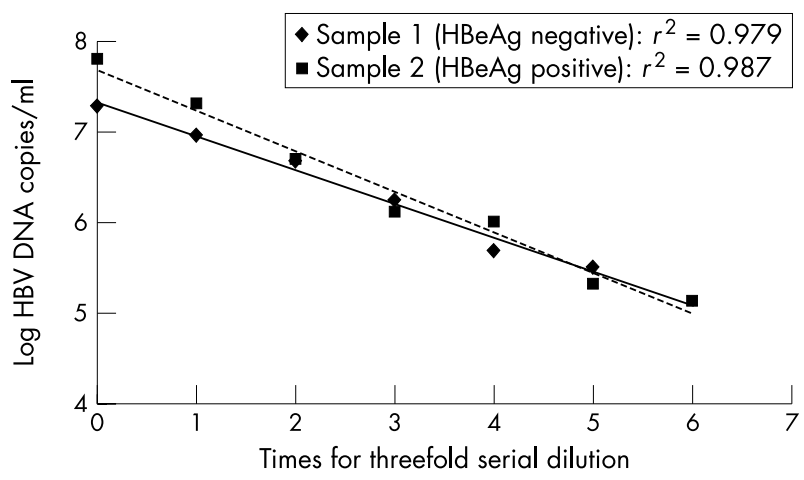

Figure 1 The linearity of hepatitis B virus (HBV) DNA measurement by the COBAS-AM test. Threefold serial dilution panels of sera from patients who were negative (sample 1) and positive (sample 2) for HBV e antigen $(\mathrm{HBeAg})$ had $2.0 \times 10^{7}$ and $6.17 \times 10^{7}$ copies of HBV DNA $/ \mathrm{ml}$, respectively. age, or HBeAg status) was the only factor associated with the ALT concentration $(p=0.01)$. Using multiple logistic regression, the serum HBV DNA value was the only factor that was significantly associated with HBeAg status (odds ratio, 2.69; $95 \%$ confidence interval, 1.80 to 4.04 ) and abnormal ALT concentration (odds ratio, 1.71; 95\% confidence interval, 1.20 to 2.43 ).

\section{DISCUSSION}

The COBAS-AM assay is a PCR based, automated assay developed for the measurement of HBV DNA in serum. In previous studies by Noborg and colleagues ${ }^{22}$ and Lopez et al, ${ }^{23}$ the assay was found to be reproducible, with a high degree of accuracy and precision in both intra-assay and interassay comparisons. In our present study on Taiwanese patients with chronic HBV, the COBAS-AM assay demonstrated a high linearity and reproducibility in the measurement of serum HBV DNA values.

The bDNA assay is a sensitive method for HBV measurement. Using the lower limit of $0.7 \mathrm{Meq} / \mathrm{ml}$, the specificity of the bDNA assay was $99.7 \%{ }^{8}$ The correlation between the bDNA assay and a crosslinking assay (NAXCOR XLnt; NAXCOR, Menlo Park, California, USA), a hybrid capture amplification assay (Digene Diagnostics, Silver Spring, Maryland, USA), and the Amplicor HBV monitor test (Roche Diagnostic Systems, Branchburg, New Jersey, USA) has been studied. ${ }^{24-28}$ Our present study has shown a high degree of correlation between the COBAS-AM test and the

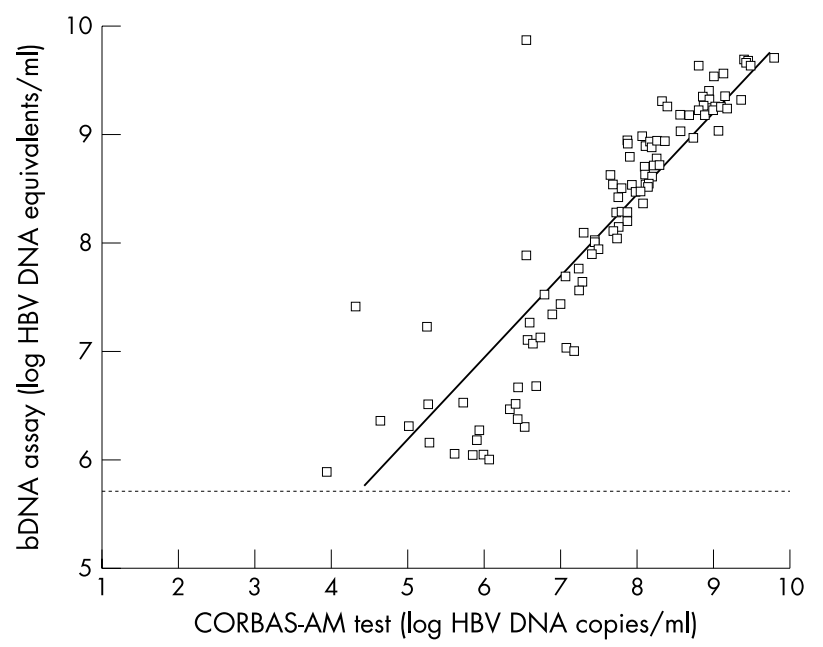

Figure 2 Comparison between hepatitis B virus (HBV) DNA values in samples from 124 patients with chronic $\mathrm{HBV}$ infection measured by the COBAS-AM test and the bDNA assay. There was a good correlation between all the specimens yielding values within the quantitative range of each of the assays $(b=1.004 ; r=0.901)$. HBV values were expressed as log equivalents or log copies $/ \mathrm{ml}$. The lower detection limit of the bDNA assay is indicated by the broken line $\left(0.7 \times 10^{6}\right.$ equivalents $/ \mathrm{ml}$, which corresponds to $5.8451 \mathrm{log}$ equivalents $/ \mathrm{ml}$ ). 
Table 2 Correlation between the results of the COBAS-AM and branched DNA assays in 124 samples positive for both

\begin{tabular}{|c|c|c|c|c|c|c|}
\hline & \multirow[b]{2}{*}{ No. } & \multicolumn{3}{|c|}{ HBV DNA value* } & \multirow{2}{*}{$\begin{array}{l}\text { Spearman's rank } \\
\text { correlation coefficient }\end{array}$} & \multirow[b]{2}{*}{ p Value } \\
\hline & & bDNA assay & COBAS-AM test & Differencet & & \\
\hline $\begin{array}{l}\text { All } \\
\mathrm{HBeAg}\end{array}$ & 124 & $8.17(1.13)$ & $7.62(1.25)$ & $0.55(0.54)$ & 0.901 & $<0.0001$ \\
\hline Positive & 76 & $8.63(0.97)$ & $8.04(1.19)$ & $0.52(0.58)$ & 0.906 & $<0.0001$ \\
\hline Negative & 48 & $7.48(0.93)$ & $6.96(1.01)$ & $0.92(0.75)$ & 0.824 & $<0.0001$ \\
\hline
\end{tabular}

bDNA assay. Gilbert et al described a comparative analysis of the performances of three assays: the bDNA assay, the Digene hybrid capture assay, and the Amplicor HBV monitor test. When using the Eurohep HBV DNA standard controls, the bDNA assay consistently over-reported by $0.5 \mathrm{log}$, compared with the Amplicor HBV monitor assay. ${ }^{28}$ In our study, we found that the values derived from the bDNA assay were on average $0.55 \log$ higher than those derived from the COBASAM test, an automated assay.

The COBAS-AM assay extended the linear range of detection for HBV DNA from $10^{5}$ to $10^{2}$ copies $/ \mathrm{ml},{ }^{22}$ thereby improving the sensitivity so that patients in whom the virus was undetectable by the bDNA assay could be assessed. We found that HBV DNA values were higher in HBeAg positive than in HBeAg negative patients. All HBeAg positive samples that could be measured by the bDNA assay could also be measured by the COBAS-AM test. Approximately 70\% of our samples with undetectable HBV DNA using the bDNA assay were positive using the COBAS-AM test. The study of Chan et al showed that approximately half of those patients with undetectable HBV DNA values using the bDNA assay were positive for HBV DNA based on an in house PCR assay. ${ }^{29}$ Because HBeAg negative patients had lower mean HBV DNA values and a higher rate of values below the LLOD of the bDNA assay, the higher sensitivity of the COBAS-AM test makes it suitable for the accurate measurement of HBV DNA values in HBeAg negative patients. The measurement of HBV DNA is useful in the evaluation and monitoring of patients with chronic HBV infection who are receiving antiviral treatment. $^{19}$ The emergence of lamivudine resistant HBV

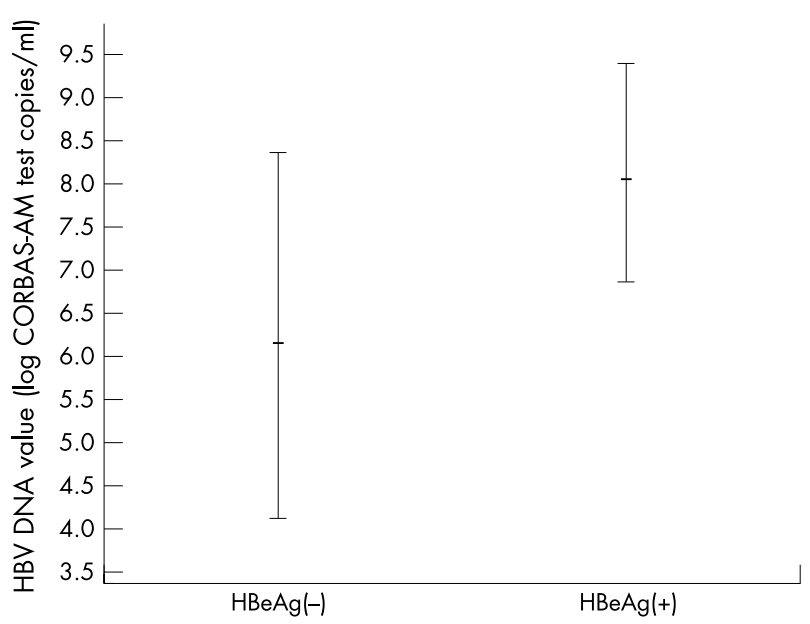

Figure 3 Comparison between hepatitis B virus (HBV) DNA values in $\mathrm{HBV}$ e antigen $(\mathrm{HBeAg})$ negative and positive samples from patients with chronic HBV infection measured by the COBAS-AM test. The HBV DNA value was significantly higher in $\mathrm{HBeAg}$ positive than in $\mathrm{HBeAg}$ negative patients $(p<0.0001$ ). The mean serum HBV DNA values and SD values are shown in each group. strains can be predicted by monitoring viral loads, using a cutoff value of $1000-10000$ copies HBV DNA/ml. ${ }^{17}{ }^{19}$ The COBAS-AM test is most useful when serum HBV DNA values are below the LLOD of the bDNA assay.

Serum ALT concentrations are mainly dependant on the different phases of the natural history of chronic HBV infection. Because most of our patients had abnormal ALT values, they represented a group of Taiwanese patients with active chronic HBV infection. This may partly explain why serum ALT concentrations and HBeAg status were independently associated with serum HBV DNA values. However, we found that the usefulness of the ALT concentration in the prediction of the HBV DNA value was questionable in our cross sectional study.

"The COBAS-AM test is most useful when serum hepatitis $b$ virus DNA values are below the lower limit of detection of the branched DNA assay"

The bDNA assay requires overnight incubation and is more time consuming than the COBAS-AM test. However, the narrower dynamic range of the COBAS-AM test (200$20000 \mathrm{copies} / \mathrm{ml}$ ) makes it necessary to predilute the serum samples and increases the costs of the tests. Noborg et al reported that the median HBV DNA value using the CORBAS-AM test for HBeAg positive samples was $10^{8.6}$ copies/ml and suggested that the most suitable predilution would be $1 / 10^{5} .{ }^{22}$ In our present study, the median HBV DNA value was $10^{7.8}$ for HBeAg positive samples, and $1 / 10^{5}$ seemed to be the most suitable predilution, with $82.1 \%$ of HBeAg positive samples being measured successfully.

In summary, the COBAS-AM assay can be used effectively to measure HBV DNA values in Taiwanese patients, and shows good linearity and reproducibility. The results of the COBAS-AM test correlated well with those of the bDNA assay. The COBAS-AM test is more sensitive among HBeAg negative samples than the bDNA assay.

\section{Take home messages}

- The COBAS-AM test, a polymerase chain reaction based assay, is more sensitive in hepatitis B virus (HBV) e antigen ( $\mathrm{HBeAg}$ ) negative samples than the branched DNA assay

- HBV DNA values measured by the COBAS-AM test and the branched DNA assay correlate significantly

- The COBAS-AM test can be used effectively to measure HBV DNA concentrations in Taiwanese patients and would be useful for monitoring treatment in chronic $\mathrm{HBV}$ infection and for assessing resistance 
Authors' affiliations

C-Y Dai, M-L Yu, S-C Chen, Z-Y Lin, M-Y Hsieh, L-Y Wang, J-F Tsai, W-L

Chuang, W-Y Chang, Hepatobiliary Division, Department of Internal Medicine, Kaohsiung Medical University, Kaohsiung 807, Taiwan

\section{REFERENCES}

1 Lee WM. Hepatitis B virus infection. N Engl J Med 1997;337:1733-45

2 Chen DS. From hepatitis to hepatoma: lessons from type B viral hepatitis. Science 1993;262:369-70.

3 Stevens C, Beasley R, Tsui J, et al. Vertical transmission of hepatitis B antigen in Taiwan. N Engl J Med 1975;292:772-4.

4 van Deursen FJ, Hino K, Wyatt D, et al. Use of PCR in resolving diagnostic difficulties potentially caused by genetic variation of hepatitis B virus. J Clin Pathol 1998:51:149-53.

5 Zhang YY, Hansson BG, Kuo LS, et al. Hepatitis B virus DNA in serum and liver is commonly found in Chinese patients with chronic liver disease despite the presence of antibodies to HBsAg. Hepatology 1993;17:538-44.

6 Cacciola I, Pollicino T, Squadrito G, et al. Occult hepatitis B virus infection in patients with chronic hepatitis C liver disease. N Engl J Med 1999;341:22-6.

7 Jardi R, Buti M, Rodriguez-Frias F, et al. The value of quantitative detection of HBV-DNA amplified by PCR in the study of hepatitis B infection. J Hepatol 1996;24:680-5

8 Hendricks DA, Stowe BJ, Hoo BS, et al. Quantitation of HBV DNA in human serum using a branched DNA (bDNA) signal amplification assay. Am J Clin Pathol 1995; 104:537-46.

9 Hoofnagle $\mathrm{JH}$, di Bisceglie AM. The treatment of chronic viral hepatitis. N Engl J Med 1997;336:347-56.

10 Barlet V, Cohard M, Thelu MA, et al. Quantitative detection of hepatitis B virus DNA in serum using chemiluminescence: comparison with radioactive solution hybridization assay. J Virol Methods 1994:49:141-51.

11 Krajden M, Minor J, Cork L, et al. Multi-measurement method comparison of three commercial hepatitis B virus DNA quantification assays. J Viral Hepat 1998;5:415-22.

12 Zaaijer HL, ter Borg F, Cuypers HT, et al. Comparison of methods for detection of hepatitis B virus DNA. J Clin Microbiol 1994;32:2088-91.

13 Janssen HL, Schoenmaker-Weber YA, Kruining H, et al. Quantitative assessment of hepatitis $B$ virus DNA in chronic hepatitis $B$ : comparison of two solution hybridization assays. J Med Virol 1993;40:307-12.

14 Pawlotsky JM, Bastie A, Lonjon I, et al. What technique should be used for routine detection and quantification of HBV DNA in clinical samples? J Virol Methods 1997;65:245-53.
15 Krajden M, Comanor L, Rifkin O et al. Assessment of hepatitis B virus DNA stability in serum by the Chiron Quantiplex branched-DNA assay. J Clin Microbiol 1998;36:382-6.

16 Butterworth LA, Prior SL, Buda PJ, et al. Comparison of four methods for quantitative measurement of hepatitis B viral DNA. J Hepatol 1996;24:686-91.

17 Buti M, Sanchez F, Cotrina M, et al. Quantitative hepatitis B virus DNA testing for the early prediction of the maintenance of response during lamivudine therapy in patients with chronic hepatitis B. J Infect Dis 2001;183:1277-80.

18 Lai CL, Chien RN, Leung NW, et al. A one-year trial of lamivudine for chronic hepatitis B. N Engl J Med 1998;339:61-8.

19 Gauthier J, Bourne EJ, Lutz MW, et al. Quantitation of hepatitis B viremia and emergence of YMDD variants in patients with chronic hepatitis $B$ treated with lamivudine. J Infect Dis 1999;180:1757-62.

20 Ranki M, Schatzl HM, Zachoval R, et al. Quantification of hepatitis B virus DNA over a wide range from serum for studying viral replicative activity in response to treatment and in recurrent infection. Hepatology 1995:21:1492-9.

21 DiDomenico N, Link $\mathrm{H}$, Knobel R, et al. COBAS AMPLICOR: fully automated RNA and DNA amplification and detection system for routine diagnostic PCR. Clin Chem 1996;42:1915-23.

22 Noborg U, Gusdal A, Pisa EK, et al. Automated quantitative analysis of hepatitis B virus DNA by using the Cobas Amplicor HBV monitor test. J Clin Microbiol 1999;37:2793-7.

23 Lopez VA, Bourne EJ, Lutz MW, et al. Assessment of the COBAS Amplicor $\mathrm{HBV}$ monitor test for quantitation of serum hepatitis B virus DNA levels. J Clin Microbiol 2002;40:1972-6.

24 Kao JH, Wood M, Chen PJ, et al. Comparison of two methods for quantification of hepatitis B viral DNA. J Gastroenterol Hepatol 1999; 14:423-6.

25 Chan HLY, Leung NWY, Lau TCM, et al. Comparison of three different sensitive assays for hepatitis $B$ virus DNA in monitoring of responses to antiviral therapy. J Clin Microbiol 2000;38:3205-8.

26 Marin IJ, Poljak M, Seme K, et al. Comparative evaluation of three commercial assays for quantitative measurement of hepatitis B virus DNA in serum samples. Hepatogastroenterology 2002;49:1390-4.

27 Ho SK, Chan TM, Cheng IK, et al. Comparison of the second-generation Digene hybrid capture assay with the branched-DNA assay for measurement of hepatitis B virus DNA in serum. J Clin Microbiol 1999;37:2461-5.

28 Gilbert N, Corden S, ljaz S, et al. Comparison of commercial assays for the quantification of HBV DNA load in health care workers: calibration differences. J Virol Methods 2002;100:37-47.

29 Chan HLY, Leung NWY, Husssain M, et al. Are pre-core HBV mutants becoming more prevalent? Hepatology 1998;28:484A 